\title{
New Robust Exponential Stability Criterion for Uncertain Neutral Systems with Discrete and Distributed Time-Varying Delays and Nonlinear Perturbations
}

\author{
Sirada Pinjai and Kanit Mukdasai \\ Department of Mathematics, Khon Kaen University, Khon Kaen 40002, Thailand \\ Correspondence should be addressed to Kanit Mukdasai, kanit@kku.ac.th \\ Received 26 April 2011; Accepted 29 June 2011 \\ Academic Editor: Martin D. Schechter \\ Copyright (C) 2011 S. Pinjai and K. Mukdasai. This is an open access article distributed under \\ the Creative Commons Attribution License, which permits unrestricted use, distribution, and \\ reproduction in any medium, provided the original work is properly cited. \\ We investigate the problem of robust exponential stability for uncertain neutral systems \\ with discrete and distributed time-varying delays and nonlinear perturbations. Based on the \\ combination of descriptor model transformation, decomposition technique of coefficient matrix, \\ and utilization of zero equation and new Lyapunov functional, sufficient conditions for robust \\ exponential stability are obtained and formulated in terms of linear matrix inequalities (LMIs) \\ The new stability conditions are less conservative and more general than some existing results.
}

\section{Introduction}

In the past decades, the problem of stability for neutral differential systems, which have delays in both its state and the derivatives of its states, has been widely investigated by many researchers. Such systems are often encountered in engineering, biology, and economics. The existence of time delay is frequently a source of instability or poor performances in the systems. Recently, some stability criteria for neutral system with time delay have been given [1-13]. The problem of delay-dependent stability for uncertain neutral systems with time-varying delays was considered [6]. Reference [13] investigated the problem of delaydependent robust stability for delay neutral type control system with time-varying structured uncertainties and time-varying delays. The problem of a new delay-dependent robust stability criterion for neutral systems was investigated. The considered system has timevarying structured uncertainties and interval time-varying delays in [7]. However, many researchers have studied the problem of stability for systems with discrete and distributed delays such as [12] which presented some stability conditions for uncertain neutral systems with discrete and distributed delays. The robust stability of uncertain linear neutral systems 
with discrete and distributed delays has been studied in [3]. In [14, 15], they studied the problem of stability for linear switching system with discrete and distributed delays. Moreover, a descriptor model transformation and a corresponding Lyapunov-Krasovskii functional have been introduced for stability analysis of systems with delays in $[10,16]$.

In this paper, we will study the problems of robust exponential stability for uncertain neutral systems with discrete and distributed time-varying delays and nonlinear perturbations. Based on a combination of descriptor model transformation, decomposition technique of coefficient matrix, and utilization of zero equation and new Lyapunov functional which improved delay-dependent stability criteria for the considered systems, sufficient conditions for the robust exponential stability are obtained and formulated in terms of linear matrix inequalities (LMIs). The new stability conditions will be less conservative and more general than some existing results.

\section{Problem Formulation and Preliminaries}

We introduce some notations and definitions that will be used throughout the paper. $\mathbb{R}^{+}$ denotes the set of all real nonnegative numbers; $\mathbb{R}^{n}$ denotes the $n$-dimensional space with the vector norm $\|\cdot\| ;\|x\|$ denotes the Euclidean vector norm of $x \in \mathbb{R}^{n} ; \mathbb{R}^{n \times r}$ denotes the set of $n \times r$ real matrices; $A^{T}$ denotes the transpose of the matrix $A ; A$ is symmetric if $A=A^{T}$; I denotes the identity matrix; $\lambda(A)$ denotes the set of all eigenvalues of $A$; $\lambda_{\max }(A)=\max \{\operatorname{Re} \lambda: \lambda \in \lambda(A)\} ; \lambda_{\min }(A)=\min \{\operatorname{Re} \lambda: \lambda \in \lambda(A)\}$; matrix $A$ is called semipositive definite $(A \geq 0)$ if $x^{T} A x \geq 0$, for all $x \in \mathbb{R}^{n} ; A$ is positive definite $(A>0)$ if $x^{T} A x>0$ for all $x \neq 0$; matrix $B$ is called seminegative definite $(B \leq 0)$ if $x^{T} B x \leq 0$, for all $x \in \mathbb{R}^{n} ; B$ is negative definite $(B<0)$ if $x^{T} B x<0$ for all $x \neq 0 ; A>B$ means $A-B>0 ; A \geq B$ means $A-B \geq 0 ; C\left([-h, 0], \mathbb{R}^{n}\right)$ denotes the space of all continuous vector functions mapping $[-h, 0]$ into $\mathbb{R}^{n} ; *$ represents the elements below the main diagonal of a symmetric matrix.

Consider the uncertain neutral system with discrete and distributed time-varying delays and nonlinear perturbations of the form

$$
\begin{aligned}
\dot{x}(t)-C \dot{x}(t-r(t))= & A(t) x(t)+B(t) x(t-h(t))+f_{1}(t, x(t)) \\
& +f_{2}(t, x(t-h(t)))+D(t) \int_{t-g(t)}^{t} x(s) d s, \quad t \geq 0, \\
x\left(t_{0}+\theta\right)= & \phi(\theta), \quad \dot{x}\left(t_{0}+\theta\right)=\varphi(\theta), \quad \forall \theta \in[-\max \{r, h, g\}, 0], \\
A(t)= & {[A+\Delta A(t)], \quad B(t)=[B+\Delta B(t)], \quad D(t)=[D+\Delta D(t)], }
\end{aligned}
$$

where $x(t) \in \mathbb{R}^{n}$ is the state, $A, B, C, D \in \mathbb{R}^{n \times n}$ are known real constant matrices. $r(t), h(t)$, and $g(t)$ are neutral, discrete, and distributed time-varying delays, respectively,

$$
\begin{gathered}
0 \leq r(t) \leq r, \quad \dot{r}(t) \leq \delta<1, \\
0 \leq h(t) \leq h, \quad \dot{h}(t) \leq \mu<+\infty, \\
0 \leq g(t) \leq g,
\end{gathered}
$$


where $h, r, g, \mu$, and $\delta$ are given positive constants. The initial condition functions $\phi(t)$, $\varphi(t) \in C\left([-\max \{r, h, g\}, 0], \mathbb{R}^{n}\right)$ denote continuous vector-valued initial function of $t \in$ $[-\max \{r, h, g\}, 0]$ with the norm $\|\phi\|=\sup _{s \in[-\max \{r, h, g\}, 0]}\|\phi(s)\|,\|\varphi\|=\sup _{s \in[-\max \{r, h, g\}, 0]}\|\varphi(s)\|$. The uncertain matrices $\Delta A(t), \Delta B(t)$, and $\Delta D(t)$ are norm bounded and can be described as

$$
\Delta A(t)=E F(t) N_{1}, \quad \Delta B(t)=E F(t) N_{2}, \quad \Delta D(t)=E F(t) N_{3},
$$

where $E, N_{i}, i=1,2,3$, are known constant matrices with appropriate dimensions. The uncertain matrix $F(t)$ satisfies

$$
F(t)^{T} F(t) \leq I
$$

The uncertainties $f_{1}(\cdot)$ and $f_{2}(\cdot)$ represent the nonlinear parameter perturbations with respect to the current state $x(t)$ and the delayed state $x(t-h(t))$, respectively, and are bounded in magnitude:

$$
\begin{gathered}
f_{1}^{T}(t, x(t)) f_{1}(t, x(t)) \leq \beta_{1} x^{T}(t) x(t), \\
f_{2}^{T}(t, x(t-h(t))) f_{2}(t, x(t-h(t))) \leq \beta_{2} x^{T}(t-h(t)) x(t-h(t)),
\end{gathered}
$$

where $\eta, \rho$ are given positive constants. First, we consider nominal system (2.1) which is defined to be

$$
\begin{aligned}
\dot{x}(t)-C \dot{x}(t-r(t))= & A x(t)+B x(t-h(t))+f_{1}(t, x(t))+f_{2}(t, x(t-h(t))) \\
& +D \int_{t-g(t)}^{t} x(s) d s, \quad t \geq 0, \\
x\left(t_{0}+\theta\right)= & \phi(\theta), \quad \dot{x}\left(t_{0}+\theta\right)=\varphi(\theta), \quad \forall \theta \in[-\max \{r, h, g\}, 0] .
\end{aligned}
$$

Rewrite the system (2.6) in the following descriptor system:

$$
\begin{aligned}
\dot{x}(t)= & y(t), \\
y(t)= & A x(t)+B x(t-h(t))+f_{1}(t, x(t))+f_{2}(t, x(t-h(t))) \\
& +C y(t-r(t))+D \int_{t-g(t)}^{t} x(s) d s .
\end{aligned}
$$


In order to improve the bound of the discrete delay $h(t)$ in (2.6), let us decompose the constant matrix $B$ as

$$
B=B_{1}+B_{2}
$$

where $B_{1}, B_{2} \in \mathbb{R}^{n \times n}$ are constant matrices. Utilize the following zero equation:

$$
0=G x(t)-G x(t-h(t))-G \int_{t-h(t)}^{t} \dot{x}(s) d s
$$

where $G \in \mathbb{R}^{n \times n}$ will be chosen to guarantee the exponential stability of the system (2.6). By (2.8) and (2.9), the system (2.7) can be represented in the form of a descriptor system with discrete and distributed delays and nonlinear perturbations:

$$
\begin{aligned}
\dot{x}(t)= & y(t)+G x(t)-G x(t-h(t))-G \int_{t-h(t)}^{t} y(s) d s \\
y(t)= & A_{1} x(t)+B_{2} x(t-h(t))+f_{1}(t, x(t))+f_{2}(t, x(t-h(t))) \\
& +C y(t-r(t))-B_{1} \int_{t-h(t)}^{t} y(s) d s+D \int_{t-g(t)}^{t} x(s) d s
\end{aligned}
$$

where $A_{1}=A+B_{1}$.

Definition 2.1. The system (2.1) is robustly exponentially stable if there exist real scalars $\alpha>0$, $M>0$ such that, for each $\phi(t) \in C\left([-\max \{r, h, g\}, 0], \mathbb{R}^{n}\right)$, the solution $x(t, \phi, \varphi)$ of the system (2.1) satisfies

$$
\|x(t, \phi, \varphi)\| \leq M \max [\|\phi\|,\|\varphi\|] e^{-\alpha t}, \quad \forall t \in \mathbb{R}^{+} .
$$

Lemma 2.2 (Jensen's inequality [17]). For any constant symmetric matrix $R>0$, scalar $h>0$, and vector function $\dot{x}(t):[-h, 0] \rightarrow \mathbb{R}^{n}$ such that the following integral is well defined, one has

$$
-h \int_{t-h}^{t} \dot{x}^{T}(s) R \dot{x}(s) d s \leq\left[\begin{array}{c}
x(t) \\
x(t-h)
\end{array}\right]^{T}\left[\begin{array}{cc}
-R & R \\
R & -R
\end{array}\right]\left[\begin{array}{c}
x(t) \\
x(t-h)
\end{array}\right] .
$$

\section{Exponential Stability Conditions}

In this section, we first study the exponential stability criteria for the nominal system (2.1) by using the combination of linear matrix inequality (LMI) technique and Lyapunov method. 
Assumption 1. All the eigenvalues of matrix $C$ are inside the unit circle.

Theorem 3.1. Under Assumption 1 and given positive constants $h, r, g, \mu, \delta, \alpha, \beta_{1}$, and $\beta_{2}$, the nominal system (2.1) is exponentially stable if there exist positive definite matrices $P_{i}, i=1,2, \ldots, 6$, any appropriate dimensional matrices $W_{1}, Q_{1}, Q_{2}, M_{i}, i=1,2, \ldots, 8$, and positive constants $\epsilon_{1}, \epsilon_{2}$ satisfying the following LMI:

$$
\Omega=\left[\begin{array}{cccccccc}
\Omega_{11} & \Omega_{12} & \Omega_{13} & \Omega_{14} & \Omega_{15} & \Omega_{16} & \Omega_{17} & \Omega_{18} \\
* & \Omega_{22} & \Omega_{23} & \Omega_{24} & Q_{2}^{T} & Q_{2}^{T} & Q_{2}^{T} C & Q_{2}^{T} D \\
* & * & \Omega_{33} & \Omega_{34} & -M_{5} & -M_{6} & -M_{7} & -M_{8} \\
* & * & * & \Omega_{44} & -M_{5} & -M_{6} & -M_{7} & -M_{8} \\
* & * & * & * & -\epsilon_{1} I & 0 & 0 & 0 \\
* & * & * & * & * & -\epsilon_{2} I & 0 & 0 \\
* & * & * & * & * & * & \Omega_{77} & 0 \\
* & * & * & * & * & * & * & \Omega_{88}
\end{array}\right]<0,
$$

where $\Omega_{11}=W_{1}+W_{1}^{T}+Q_{1}^{T} A_{1}+A_{1}^{T} Q_{1}+P_{2}-e^{-2 \alpha h} P_{4}+g^{2} P_{6}+M_{1}^{T}+M_{1}+\epsilon_{1} \beta_{1}^{2} I+2 \alpha P_{1}, \Omega_{22}=$ $-Q_{2}-Q_{2}^{T}+h^{2} P_{3}+h^{2} P_{4}+P_{5}, \Omega_{23}=Q_{2}^{T} B_{2}-M_{2}^{T}, \Omega_{24}=Q_{2}^{T} B_{1}-M_{2}^{T}, \Omega_{33}=-e^{-2 \alpha h} P_{2}+\mu P_{2}-e^{-2 \alpha h} P_{4}-$ $M_{3}^{T}-M_{3}+\epsilon_{2} \beta_{2}^{2} I, \Omega_{34}=-M_{3}^{T}-M_{4}, \Omega_{44}=-e^{-2 \alpha h} P_{3}-M_{4}-M_{4}^{T}, \Omega_{77}=-(1-\delta) e^{-2 \alpha r} P_{5}, \Omega_{88}=$ $-e^{-2 \alpha g} P_{6}, \Omega_{12}=P_{1}-Q_{1}^{T}+A_{1}^{T} Q_{2}+M_{2}, \Omega_{13}=-W_{1}+Q_{1}^{T} B_{2}+e^{-2 \alpha h} P_{4}-M_{1}^{T}+M_{3}, \Omega_{14}=$ $-W_{1}-Q_{1}^{T} B_{1}-M_{1}^{T}+M_{4}, \Omega_{15}=Q_{1}^{T}+M_{5}, \Omega_{16}=Q_{1}^{T}+M_{6}, \Omega_{17}=Q_{1}^{T} C+M_{7}, \Omega_{18}=Q_{1}^{T} D+M_{8}$.

Moreover, the solution $x(t, \phi, \varphi)$ satisfies the inequality

$$
\|x(t, \phi, \varphi)\| \leq \sqrt{\frac{N}{\lambda_{\min }\left(P_{1}\right)}} \max [\|\phi\|,\|\varphi\|] e^{-\alpha t}, \quad t \in \mathbb{R}^{+},
$$

where

$$
N=\lambda_{\max }\left(P_{1}\right)+h \lambda_{\max }\left(P_{2}\right)+h^{3} \lambda_{\max }\left(P_{3}\right)+h^{3} \lambda_{\max }\left(P_{4}\right)+r \lambda_{\max }\left(P_{5}\right)+g^{3} \lambda_{\max }\left(P_{6}\right) .
$$

Proof. Construct a Lyapunov functional candidate for the nominal system (2.1) of the form

$$
V(t)=\sum_{i=1}^{6} V_{i}(t)
$$


where

$$
\begin{aligned}
& V_{1}(t)=x^{T}(t) P_{1} x(t)=\left[\begin{array}{l}
x(t) \\
y(t)
\end{array}\right]^{T}\left[\begin{array}{ll}
I & 0 \\
0 & 0
\end{array}\right]\left[\begin{array}{ll}
P_{1} & 0 \\
Q_{1} & Q_{2}
\end{array}\right]\left[\begin{array}{l}
x(t) \\
y(t)
\end{array}\right], \\
& V_{2}(t)=\int_{t-h(t)}^{t} e^{2 \alpha(s-t)} x^{T}(s) P_{2} x(s) d s, \\
& V_{3}(t)=h \int_{-h}^{0} \int_{t+s}^{t} e^{2 \alpha(s-t)} y^{T}(\theta) P_{3} y(\theta) d \theta d s, \\
& V_{4}(t)=h \int_{-h}^{0} \int_{t+s}^{t} e^{2 \alpha(s-t)} y^{T}(\theta) P_{4} y(\theta) d \theta d s, \\
& V_{5}(t)=\int_{t-r(t)}^{t} e^{2 \alpha(s-t)} y^{T}(s) P_{5} y(s) d s, \\
& V_{6}(t)=g \int_{-g}^{0} \int_{t+s}^{t} e^{2 \alpha(s-t)} x^{T}(\theta) P_{6} x(\theta) d \theta d s .
\end{aligned}
$$

The derivative of $V(\cdot)$ along the trajectories of nominal system (2.1) is given by

$$
\dot{V}(t)=\sum_{i=1}^{6} \dot{V}_{i}(t)
$$

where

$$
\begin{aligned}
\dot{V}_{1}(t)= & 2\left[\begin{array}{l}
x(t) \\
y(t)
\end{array}\right]^{T}\left[\begin{array}{cc}
P_{1} & Q_{1}^{T} \\
0 & Q_{2}^{T}
\end{array}\right]\left[\begin{array}{c}
\dot{x}(t) \\
0
\end{array}\right] \\
= & 2 x^{T}(t) P_{1}\left[y(t)+G x(t)-G x(t-h(t))-G \int_{t-h(t)}^{t} y(s) d s\right] \\
& +2 x^{T}(t) Q_{1}^{T}\left[-y(t)+A_{1} x(t)+B_{2} x(t-h(t))+f_{1}(t, x(t))\right. \\
& \left.\quad+f_{2}(t, x(t-h(t)))+C y(t-r(t))-B_{1} \int_{t-h(t)}^{t} y(s) d s+D \int_{t-g(t)}^{t} x(s) d s\right] \\
& +2 y^{T}(t) Q_{2}^{T}\left[-y(t)+A_{1} x(t)+B_{2} x(t-h(t))+f_{1}(t, x(t))+f_{2}(t, x(t-h(t)))\right. \\
& \left.+C y(t-r(t))-B_{1} \int_{t-h(t)}^{t} y(s) d s+D \int_{t-g(t)}^{t} x(s) d s\right] \\
& +2 \alpha x^{T}(t) P_{1} x(t)-2 \alpha V_{1}(t), \\
\dot{V}_{2}(t)= & x^{T}(t) P_{2} x(t)-\left(1-\dot{h}_{(t)}\right) e^{-2 \alpha h(t)} x^{T}(t-h(t)) P_{2} x(t-h(t))-2 \alpha V_{2}(t) \\
\leq & x^{T}(t) P_{2} x(t)-e^{-2 \alpha h} x^{T}(t-h(t)) P_{2} x(t-h(t))+\mu x^{T}(t-h(t)) P_{2} x(t-h(t))-2 \alpha V_{2}(t) .
\end{aligned}
$$


Obviously, for any scalar $s \in[t-h, t]$, we get $e^{-2 \alpha h} \leq e^{-2 \alpha(s-t)} \leq 1$. Together with Lemma 2.2 (Jensen's inequality), we obtain

$$
\begin{aligned}
& \dot{V}_{3}(t)=h^{2} y^{T}(t) P_{3} y(t)-h \int_{-h}^{0} e^{2 \alpha s} y^{T}(t+s) P_{3} y(t+s) d s-2 \alpha V_{3}(t) \\
& \leq h^{2} y^{T}(t) P_{3} y(t)-h \int_{t-h}^{t} e^{2 \alpha(s-t)} y^{T}(s) P_{3} y(s) d s-2 \alpha V_{3}(t) \\
& \leq h^{2} y^{T}(t) P_{3} y(t)-h e^{-2 \alpha h} \int_{t-h}^{t} y^{T}(s) P_{3} y(s) d s-2 \alpha V_{3}(t) \\
& \leq h^{2} y^{T}(t) P_{3} y(t)-e^{-2 \alpha h} \int_{t-h}^{t} y^{T}(s) d s P_{3} \int_{t-h}^{t} y(s) d s-2 \alpha V_{3}(t) \\
& \leq h^{2} y^{T}(t) P_{3} y(t)-e^{-2 \alpha h} \int_{t-h(t)}^{t} y^{T}(s) d s P_{3} \int_{t-h(t)}^{t} y(s) d s-2 \alpha V_{3}(t), \\
& \dot{V}_{4}(t) \leq h^{2} y^{T}(t) P_{4} y(t)-e^{-2 \alpha h} \int_{t-h(t)}^{t} y^{T}(s) d s P_{4} \int_{t-h(t)}^{t} y(s) d s-2 \alpha V_{4}(t) \\
& =h^{2} y^{T}(t) P_{4} y(t)-e^{-2 \alpha h}\left[x^{T}(t)-x^{T}(t-h(t))\right] P_{4}[x(t)-x(t-h(t))]-2 \alpha V_{4}(t), \\
& \dot{V}_{5}(t)=x^{T}(t) P_{5} x(t)-(1-\dot{r}(t)) e^{-2 \alpha r(t)} x^{T}(t-r(t)) P_{2} x(t-r(t))-2 \alpha V_{5}(t) \\
& \leq x^{T}(t) P_{5} x(t)-(1-\delta) e^{-2 \alpha r} x^{T}(t-r(t)) P_{5} x(t-r(t))-2 \alpha V_{5}(t), \\
& \dot{V}_{6}(t) \leq g^{2} x^{T}(t) P_{6} x(t)-e^{-2 \alpha g} \int_{t-g(t)}^{t} x^{T}(s) d s P_{6} \int_{t-g(t)}^{t} x(s) d s-2 \alpha V_{6}(t) .
\end{aligned}
$$

From the Leibniz-Newton formula, the following equation is true for any real matrices $M_{i}, i=1,2, \ldots, 8$, with appropriate dimensions

$$
\begin{aligned}
& 2\left[x^{T}(t) M_{1}^{T}+y^{T}(t) M_{2}^{T}+x^{T}(t-h(t)) M_{3}^{T}+\int_{t-h(t)}^{t} y^{T}(s) d s M_{4}\right. \\
& \left.+f_{1}^{T}(t, x(t)) M_{5}+f_{2}^{T}(t, x(t-h(t))) M_{6}^{T}+y^{T}(t-r(t)) M_{7}^{T}+\int_{t-g(t)}^{t} x^{T}(s) d s M_{8}\right] \\
& \quad \times\left[x(t)-x(t-h(t))-\int_{t-h(t)}^{t} y(s) d s\right]=0 .
\end{aligned}
$$


From (2.5), we obtain for any scalars $\epsilon_{1}, \epsilon_{2}>0$

$$
\begin{gathered}
0 \leq \epsilon_{1} \beta_{1} x^{T}(t) x(t)-\epsilon_{1} f_{1}^{T}(t, x(t)) f_{1}(t, x(t)), \\
0 \leq \epsilon_{2} \beta_{2} x^{T}(t-h(t)) x(t-h(t))-\epsilon_{2} f_{2}^{T}(t, x(t-h(t))) f_{2}(t, x(t-h(t))) .
\end{gathered}
$$

Let us define $W_{1}=P_{1} G$. From (3.6), (3.9), and (3.10), we obtain

$$
\dot{V}(t)+2 \alpha V(t) \leq \xi^{T}(t) \Omega \xi(t)
$$

where $\xi^{T}(t)=\left[x^{T}(t), y^{T}(t), x^{T}(t-h(t)), \int_{t-h(t)}^{t} y^{T}(s) d s, f^{T}(t, x(t)), g^{T}(t, x(t-h(t))), y^{T}(t-\right.$ $\left.r(t)), \int_{t-g(t)}^{t} x^{T}(s) d s\right]$ and

$$
\Omega=\left[\begin{array}{cccccccc}
\Omega_{11} & \Omega_{12} & \Omega_{13} & \Omega_{14} & \Omega_{15} & \Omega_{16} & \Omega_{17} & \Omega_{18} \\
* & \Omega_{22} & \Omega_{23} & \Omega_{24} & Q_{2}^{T} & Q_{2}^{T} & Q_{2}^{T} C & Q_{2}^{T} D \\
* & * & \Omega_{33} & \Omega_{34} & -M_{5} & -M_{6} & -M_{7} & -M_{8} \\
* & * & * & \Omega_{44} & -M_{5} & -M_{6} & -M_{7} & -M_{8} \\
* & * & * & * & -\epsilon_{1} I & 0 & 0 & 0 \\
* & * & * & * & * & -\epsilon_{2} I & 0 & 0 \\
* & * & * & * & * & * & \Omega_{77} & 0 \\
* & * & * & * & * & * & * & \Omega_{88}
\end{array}\right] .
$$

It is a fact that, if $\Omega<0$, then

$$
\dot{V}(t)+2 \alpha V(t) \leq 0, \quad \forall t \in \mathbb{R}^{+},
$$

which gives

$$
V(t) \leq V(0) e^{-2 \alpha t}, \quad \forall t \in \mathbb{R}^{+}
$$

From (3.14), it is easy to see that

$$
\begin{gathered}
\lambda_{\min }\left(P_{1}\right)\|x(t)\|^{2} \leq V(t) \leq V(0) e^{-2 \alpha t}, \\
V(0)=\sum_{i=1}^{6} V_{i}(0),
\end{gathered}
$$


where

$$
\begin{aligned}
& V_{1}(0)=x^{T}(0) P_{1} x(0), \\
& V_{2}(0)=\int_{-h(0)}^{0} e^{2 \alpha s} x^{T}(s) P_{2} x(s) d s, \\
& V_{3}(0)=h \int_{-h}^{0} \int_{s}^{0} e^{2 \alpha s} y^{T}(\theta) P_{3} y(\theta) d \theta d s, \\
& V_{4}(0)=h \int_{-h}^{0} \int_{s}^{0} e^{2 \alpha s} y^{T}(\theta) P_{4} y(\theta) d \theta d s, \\
& V_{5}(0)=\int_{-r(0)}^{0} e^{2 \alpha s} y^{T}(s) P_{5} y(s) d s, \\
& V_{6}(0)=g \int_{-g}^{0} \int_{s}^{0} e^{2 \alpha s} x^{T}(\theta) P_{6} x(\theta) d \theta d s .
\end{aligned}
$$

Therefore, we get

$$
\lambda_{\min }\left(P_{1}\right)\|x(t)\|^{2} \leq V(0) e^{-2 \alpha t} \leq N \max [\|\phi\|,\|\varphi\|]^{2} e^{-2 \alpha t},
$$

where $N=\lambda_{\max }\left(P_{1}\right)+h \lambda_{\max }\left(P_{2}\right)+h^{3} \lambda_{\max }\left(P_{3}\right)+h^{3} \lambda_{\max }\left(P_{4}\right)+r \lambda_{\max }\left(P_{5}\right)+g^{3} \lambda_{\max }\left(P_{6}\right)$. From (3.17), we get

$$
\|x(t, \phi, \varphi)\| \leq \sqrt{\frac{N}{\lambda_{\min }\left(P_{1}\right)}} \max [\|\phi\|,\|\varphi\|] e^{-\alpha t}, \quad \forall t \in \mathbb{R}^{+} .
$$

This means that the system (2.1) is exponentially stable. The proof of the theorem is complete.

$$
\text { If } C=\Delta A(t)=\Delta B(t)=f_{1}(t, x(t))=f_{2}(t, x(t-h(t)))=D(t)=0 \text {, then system }(2.1)
$$
reduces to the following system:

$$
\begin{gathered}
\dot{x}(t)=A x(t)+B x(t-h(t)), \quad t \geq 0, \\
x\left(t_{0}+\theta\right)=\phi(\theta), \quad \dot{x}\left(t_{0}+\theta\right)=\varphi(\theta), \quad \forall \theta \in[-h, 0] .
\end{gathered}
$$


Take the Lyapunov functional as

$$
\dot{V}(t)=\sum_{i=1}^{4} \dot{V}_{i}(t)
$$

where $V_{1}(t), V_{2}(t), V_{3}(t)$, and $V_{4}(t)$ are defined in Theorem 3.1. According to Theorem 3.1, we have the following corollary for the delay-dependent exponential stability criterion of system (3.19).

Corollary 3.2. For given positive constants $h, \mu$, and $\alpha$, the system (3.19) is exponentially stable if there exist positive definite matrices $P_{i}, i=1,2, \ldots, 4$, and any appropriate dimensional matrices $W_{1}, Q_{1}, Q_{2}, M_{i}, i=1,2, \ldots, 4$, satisfying the following $L M I$ :

$$
\Phi=\left[\begin{array}{cccc}
\Phi_{11} & \Phi_{12} & \Phi_{13} & \Phi_{14} \\
* & \Phi_{22} & \Phi_{23} & \Phi_{24} \\
* & * & \Phi_{33} & \Phi_{34} \\
* & * & * & \Phi_{44}
\end{array}\right]<0,
$$

where $\Phi_{11}=W_{1}+W_{1}^{T}+Q_{1}^{T} A_{1}+A_{1}^{T} Q_{1}+P_{2}-e^{-2 \alpha h} P_{4}+M_{1}^{T}+M_{1}+2 \alpha P_{1}, \Phi_{22}=-Q_{2}-Q_{2}^{T}+h^{2} P_{3}+$ $h^{2} P_{4}, \Phi_{23}=Q_{2}^{T} B_{2}-M_{2}^{T}, \Phi_{24}=Q_{2}^{T} B_{1}-M_{2}^{T}, \Phi_{33}=-e^{-2 \alpha h} P_{2}+\mu P_{2}-e^{-2 \alpha h} P_{4}-M_{3}^{T}-M_{3}, \Omega_{34}=$ $-M_{3}^{T}-M_{4}, \Phi_{44}=-e^{-2 \alpha h} P_{3}-M_{4}-M_{4}^{T}, \Phi_{12}=P_{1}-Q_{1}^{T}+A_{1}^{T} Q_{2}+M_{2}, \Phi_{13}=-W_{1}+Q_{1}^{T} B_{2}+$ $e^{-2 \alpha h} P_{4}-M_{1}^{T}+M_{3}, \Phi_{14}=-W_{1}-Q_{1}^{T} B_{1}-M_{1}^{T}+M_{4}$.

Moreover, the solution $x(t, \phi, \varphi)$ satisfies the inequality

$$
\|x(t, \phi, \varphi)\| \leq \sqrt{\frac{K}{\lambda_{\min }\left(P_{1}\right)}} \max [\|\phi\|,\|\varphi\|] e^{-\alpha t}, \quad t \in \mathbb{R}^{+},
$$

where

$$
K=\lambda_{\max }\left(P_{1}\right)+h \lambda_{\max }\left(P_{2}\right)+h^{3} \lambda_{\max }\left(P_{3}\right)+h^{3} \lambda_{\max }\left(P_{4}\right) .
$$

If $\Delta A(t)=\Delta B(t)=D(t)=0$, then system (2.1) reduces to the following system:

$$
\begin{gathered}
\dot{x}(t)-C \dot{x}(t-r(t))=A x(t)+B x(t-h(t))+f_{1}(t, x(t))+f_{2}(t, x(t-h(t))), \quad t \geq 0, \\
x\left(t_{0}+\theta\right)=\phi(\theta), \quad \dot{x}\left(t_{0}+\theta\right)=\varphi(\theta), \quad \forall \theta \in[-\max \{r, h\}, 0] .
\end{gathered}
$$


Take the Lyapunov functional as

$$
\dot{V}(t)=\sum_{i=1}^{5} \dot{V}_{i}(t)
$$

where $V_{1}(t), V_{2}(t), V_{3}(t), V_{4}(t)$, and $V_{5}(t)$ are defined in Theorem 3.1. According to Theorem 3.1, we have the following corollary for the delay-dependent exponential stability criterion of system (3.24).

Corollary 3.3. For given positive constants $h, r, \mu, \delta, \alpha, \beta_{1}$, and $\beta_{2}$, the system (3.24) is exponentially stable if there exist positive definite matrices $P_{i}, i=1,2, \ldots, 5$, any appropriate dimensional matrices $W_{1}, Q_{1}, Q_{2}, M_{i}, i=1,2, \ldots, 7$, and positive constants $\epsilon_{1}, \epsilon_{2}$ satisfying the following LMI:

$$
\Upsilon=\left[\begin{array}{ccccccc}
\Upsilon_{11} & \Upsilon_{12} & \Upsilon_{13} & \Upsilon_{14} & \Upsilon_{15} & \Upsilon_{16} & \Upsilon_{17} \\
* & \Upsilon_{22} & \Upsilon_{23} & \Upsilon_{24} & Q_{2}^{T} & Q_{2}^{T} & Q_{2}^{T} C \\
* & * & \Upsilon_{33} & \Upsilon_{34} & -M_{5} & -M_{6} & -M_{7} \\
* & * & * & \Upsilon_{44} & -M_{5} & -M_{6} & -M_{7} \\
* & * & * & * & -\epsilon_{1} I & 0 & 0 \\
* & * & * & * & * & -\epsilon_{2} I & 0 \\
* & * & * & * & * & * & \Upsilon_{77}
\end{array}\right]<0,
$$

where $\Upsilon_{11}=W_{1}+W_{1}^{T}+Q_{1}^{T} A_{1}+A_{1}^{T} Q_{1}+P_{2}-e^{-2 \alpha h} P_{4}+M_{1}^{T}+M_{1}+\epsilon_{1} \beta_{1}^{2} I+2 \alpha P_{1}, \Upsilon_{22}=-Q_{2}-Q_{2}^{T}+$ $h^{2} P_{3}+h^{2} P_{4}+P_{5}, \Upsilon_{23}=Q_{2}^{T} B_{2}-M_{2}^{T}, \Upsilon_{24}=Q_{2}^{T} B_{1}-M_{2}^{T}, \Upsilon_{33}=-e^{-2 \alpha h} P_{2}+\mu P_{2}-e^{-2 \alpha h} P_{4}-M_{3}^{T}-$ $M_{3}+\epsilon_{2} \beta_{2}^{2} I, \Upsilon_{34}=-M_{3}^{T}-M_{4}, \Upsilon_{44}=-e^{-2 \alpha h} P_{3}-M_{4}-M_{4}^{T}, \Upsilon_{12}=P_{1}-Q_{1}^{T}+A_{1}^{T} Q_{2}+M_{2}, \Upsilon_{13}=$ $-W_{1}+Q_{1}^{T} B_{2}+e^{-2 \alpha h} P_{4}-M_{1}^{T}+M_{3}, \Upsilon_{14}=-W_{1}-Q_{1}^{T} B_{1}-M_{1}^{T}+M_{4}, \Upsilon_{15}=Q_{1}^{T}+M_{5}, \Upsilon_{16}=$ $Q_{1}^{T}+M_{6}, \Upsilon_{17}=Q_{1}^{T} C+M_{7}, \Upsilon_{77}=-(1-\delta) e^{-2 \alpha r} P_{5}$.

Moreover, the solution $x(t, \phi, \varphi)$ satisfies the inequality

$$
\|x(t, \phi, \varphi)\| \leq \sqrt{\frac{K}{\lambda_{\min }\left(P_{1}\right)}} \max [\|\phi\|,\|\varphi\|] e^{-\alpha t}, \quad t \in \mathbb{R}^{+},
$$

where

$$
K=\lambda_{\max }\left(P_{1}\right)+h \lambda_{\max }\left(P_{2}\right)+h^{3} \lambda_{\max }\left(P_{3}\right)+h^{3} \lambda_{\max }\left(P_{4}\right) .
$$

\section{Robust Exponential Stability Conditions}

Theorem 4.1. Under Assumption 1 and given positive constants $h, r, g, \mu, \delta, \alpha, \beta_{1}$, and $\beta_{2}$, the system (2.1) is exponentially stable if there exist positive definite matrices $P_{i}, i=1,2, \ldots, 6$, any 
appropriate dimensional matrices $W_{1}, Q_{1}, Q_{2}, M_{i}, i=1,2, \ldots, 8$, and positive constants $\epsilon_{1}, \epsilon_{2}$, and $\varepsilon$ satisfying the following LMI:

$$
\tilde{\Omega}=\left[\begin{array}{cccccccccc}
\Omega_{11} & \Omega_{12} & \Omega_{13} & \Omega_{14} & \Omega_{15} & \Omega_{16} & \Omega_{17} & \Omega_{18} & Q_{1}^{T} E & \varepsilon N_{1}^{T} \\
* & \Omega_{22} & \Omega_{23} & \Omega_{24} & Q_{2}^{T} & Q_{2}^{T} & Q_{2}^{T} C & Q_{2}^{T} D & Q_{1}^{T} E & 0 \\
* & * & \Omega_{33} & \Omega_{34} & -M_{5} & -M_{6} & -M_{7} & -M_{8} & 0 & \varepsilon N_{2}^{T} \\
* & * & * & \Omega_{44} & -M_{5} & -M_{6} & -M_{7} & -M_{8} & 0 & 0 \\
* & * & * & * & -\epsilon_{1} I & 0 & 0 & 0 & 0 & 0 \\
* & * & * & * & * & -\epsilon_{2} I & 0 & 0 & 0 & 0 \\
* & * & * & * & * & * & \Omega_{77} & 0 & 0 & 0 \\
* & * & * & * & * & * & * & \Omega_{88} & 0 & \varepsilon N_{3}^{T} \\
* & * & * & * & * & * & * & * & -\varepsilon I & 0 \\
* & * & * & * & * & * & * & * & * & -\varepsilon I
\end{array}\right]<0,
$$

where $\Omega_{11}=W_{1}+W_{1}^{T}+Q_{1}^{T} A_{1}+A_{1}^{T} Q_{1}+P_{2}-e^{-2 \alpha h} P_{4}+g^{2} P_{6}+M_{1}^{T}+M_{1}+\epsilon_{1} \beta_{1}^{2} I+2 \alpha P_{1}, \Omega_{22}=$ $-Q_{2}-Q_{2}^{T}+h^{2} P_{3}+h^{2} P_{4}+P_{5}, \Omega_{23}=Q_{2}^{T} B_{2}-M_{2}^{T}, \Omega_{24}=Q_{2}^{T} B_{1}-M_{2}^{T}, \Omega_{33}=-e^{-2 \alpha h} P_{2}+\mu P_{2}-e^{-2 \alpha h} P_{4}-$ $M_{3}^{T}-M_{3}+\epsilon_{2} \beta_{2}^{2} I, \Omega_{34}=-M_{3}^{T}-M_{4}, \Omega_{44}=-e^{-2 \alpha h} P_{3}-M_{4}-M_{4}^{T}, \Omega_{77}=-(1-\delta) e^{-2 \alpha r} P_{5}, \Omega_{88}=$ $-e^{-2 \alpha g} P_{6}, \Omega_{12}=P_{1}-Q_{1}^{T}+A_{1}^{T} Q_{2}+M_{2}, \Omega_{13}=-W_{1}+Q_{1}^{T} B_{2}+e^{-2 \alpha h} P_{4}-M_{1}^{T}+M_{3}, \Omega_{14}=$ $-W_{1}-Q_{1}^{T} B_{1}-M_{1}^{T}+M_{4}, \Omega_{15}=Q_{1}^{T}+M_{5}, \Omega_{16}=Q_{1}^{T}+M_{6}, \Omega_{17}=Q_{1}^{T} C+M_{7}, \Omega_{18}=Q_{1}^{T} D+M_{8}$.

Moreover, the solution $x(t, \phi, \varphi)$ satisfies the inequality

$$
\|x(t, \phi, \varphi)\| \leq \sqrt{\frac{N}{\lambda_{\min }\left(P_{1}\right)}} \max [\|\phi\|,\|\varphi\|] e^{-\alpha t}, \quad t \in \mathbb{R}^{+},
$$

where

$$
N=\lambda_{\max }\left(P_{1}\right)+h \lambda_{\max }\left(P_{2}\right)+h^{3} \lambda_{\max }\left(P_{3}\right)+h^{3} \lambda_{\max }\left(P_{4}\right)+r \lambda_{\max }\left(P_{5}\right)+g^{3} \lambda_{\max }\left(P_{6}\right) .
$$

Proof. Replacing $A_{1}, B_{2}$, and $D$ in (3.26) with $A_{1}(t)=A+B_{1}+E F(t) N_{1}, B_{2}(t)=B_{2}+E F(t) N_{2}$, and $D(t)=D+E F(t) N_{3}$, respectively, we find that condition (3.26) for system (2.1) is equivalent to the following condition:

$$
\Omega+\Gamma F(t) \Pi+\Pi^{T} F(t)^{T} \Gamma^{T}<0,
$$

where $\Gamma^{T}=\left[E^{T} Q_{1}, E^{T} Q_{2}, 0,0,0,0,0,0\right]$ and $\Pi=\left[N_{1}, 0, N_{2}, 0,0,0,0, N_{3}\right]$. Using Lemma 1 in [9], we have that there exists a positive number $\varepsilon>0$. We can find that (4.4) is equivalent to the LMI as follows:

$$
\Omega+\varepsilon^{-1} \Gamma \Gamma^{T}+\varepsilon \Pi^{T} \Pi<0 .
$$


Applying Lemma 1 (Schur complement lemma) in [12], we obtain

$$
\left[\begin{array}{ccc}
\Omega & \Gamma & \varepsilon \Pi^{T} \\
\Gamma^{T} & -\varepsilon I & 0 \\
\varepsilon \Pi & 0 & -\varepsilon I
\end{array}\right]<0 .
$$

Hence, we conclude that (4.6) is equivalent to (4.1). The proof of the theorem is complete. system:

If $f_{1}(t, x(t))=f_{2}(t, x(t-h(t)))=D(t)=0$, then system (2.1) reduces to the following

$$
\begin{gathered}
\dot{x}(t)-C \dot{x}(t-r(r))=\left[A+E F(t) N_{1}\right] x(t)+\left[B+E F(t) N_{2}\right] x(t-h(t)), \quad t \geq 0, \\
x\left(t_{0}+\theta\right)=\phi(\theta), \quad \dot{x}\left(t_{0}+\theta\right)=\varphi(\theta), \quad \forall \theta \in[-\max \{r, h\}, 0] .
\end{gathered}
$$

Take the Lyapunov functional as

$$
\dot{V}(t)=\sum_{i=1}^{5} \dot{V}_{i}(t)
$$

where $V_{1}(t), V_{2}(t), V_{3}(t), V_{4}(t)$, and $V_{5}(t)$ are defined in Theorem 3.1. According to Theorems 3.1 and 4.1 , we have the following corollary for the delay-dependent robust exponential stability of system (4.7).

Corollary 4.2. Under Assumption 1 and given positive constants $h, r, \mu, \delta$, and $\alpha$, the system (4.7) is robustly exponentially stable if there exist positive definite matrices $P_{i}, i=1,2, \ldots, 5$, any appropriate dimensional matrices $W_{1}, Q_{1}, Q_{2}, M_{7}, M_{i}, i=1,2, \ldots, 4$, and positive constant $\varepsilon$ satisfying the following LMI:

$$
\widetilde{\Omega}=\left[\begin{array}{ccccccc}
\Omega_{11} & \Omega_{12} & \Omega_{13} & \Omega_{14} & \Omega_{17} & Q_{1}^{T} E & \varepsilon N_{1}^{T} \\
* & \Omega_{22} & \Omega_{23} & \Omega_{24} & Q_{2}^{T} C & Q_{1}^{T} E & 0 \\
* & * & \Omega_{33} & \Omega_{34} & -M_{7} & 0 & \varepsilon N_{2}^{T} \\
* & * & * & \Omega_{44} & -M_{7} & 0 & 0 \\
* & * & * & * & \Omega_{77} & 0 & 0 \\
* & * & * & * & * & -\varepsilon I & 0 \\
* & * & * & * & * & * & -\varepsilon I
\end{array}\right]<0,
$$

where $\Omega_{11}=W_{1}+W_{1}^{T}+Q_{1}^{T} A_{1}+A_{1}^{T} Q_{1}+P_{2}-e^{-2 \alpha h} P_{4}+M_{1}^{T}+M_{1}+2 \alpha P_{1}, \Omega_{22}=-Q_{2}-Q_{2}^{T}+h^{2} P_{3}+$ $h^{2} P_{4}+P_{5}, \Omega_{23}=Q_{2}^{T} B_{2}-M_{2}^{T}, \Omega_{24}=Q_{2}^{T} B_{1}-M_{2}^{T}, \Omega_{33}=-e^{-2 \alpha h} P_{2}+\mu P_{2}-e^{-2 \alpha h} P_{4}-M_{3}^{T}-M_{3}, \Omega_{34}=$ $-M_{3}^{T}-M_{4}, \Omega_{44}=-e^{-2 \alpha h} P_{3}-M_{4}-M_{4}^{T}, \Omega_{77}=-(1-\delta) e^{-2 \alpha r} P_{5}, \Omega_{12}=P_{1}-Q_{1}^{T}+A_{1}^{T} Q_{2}+M_{2}, \Omega_{13}=$ $-W_{1}+Q_{1}^{T} B_{2}+e^{-2 \alpha h} P_{4}-M_{1}^{T}+M_{3}, \Omega_{14}=-W_{1}-Q_{1}^{T} B_{1}-M_{1}^{T}+M_{4}, \Omega_{17}=Q_{1}^{T} C+M_{7}$. 
Table 1: Comparison of the maximum allowed time delay $h_{\max }$.

\begin{tabular}{lcccc}
\hline Methods & $h_{\max }(\delta=0.9)$ & $h_{\max }(\delta=1)$ & $h_{\max }(\delta=1.2)$ & $h_{\max }(\delta=1.5)$ \\
\hline Fridman and Shaked [16] & 1.18 & 0.99 & 0.99 & 0.99 \\
He et al. [4] & 1.37 & 1.34 & 1.34 & 1.34 \\
Zhu and Yang [18] & 1.43 & 1.39 & 1.36 & 1.35 \\
Our results & 2.00 & 1.51 & 1.51 & 1.51 \\
\hline
\end{tabular}

Moreover, the solution $x(t, \phi, \varphi)$ satisfies the inequality

$$
\|x(t, \phi, \varphi)\| \leq \sqrt{\frac{Z}{\lambda_{\min }\left(P_{1}\right)}} \max [\|\phi\|,\|\varphi\|] e^{-\alpha t}, \quad t \in \mathbb{R}^{+},
$$

where

$$
Z=\lambda_{\max }\left(P_{1}\right)+h \lambda_{\max }\left(P_{2}\right)+h^{3} \lambda_{\max }\left(P_{3}\right)+h^{3} \lambda_{\max }\left(P_{4}\right)+r \lambda_{\max }\left(P_{5}\right) .
$$

\section{Numerical Examples}

In order to show the effectiveness of the approaches presented in Sections 3 and 4, three numerical examples are provided.

Example 5.1. Consider the asymptotic stability $(\alpha=0)$ of the linear system (3.19) with timevarying delays when

$$
A=\left[\begin{array}{cc}
-2 & 0 \\
0 & -0.9
\end{array}\right], \quad B=\left[\begin{array}{cc}
-1 & 0 \\
-1 & -1
\end{array}\right] .
$$

Decompose matrix $B$ as follows: $B=B_{1}+B_{2}$, where

$$
B_{1}=\left[\begin{array}{cc}
-0.83 & 0 \\
-1 & -0.83
\end{array}\right], \quad B_{2}=\left[\begin{array}{cc}
-0.17 & 0 \\
0 & -0.17
\end{array}\right]
$$

Table 1 lists the comparison of the upper bounds delays for asymptotic stability $(\alpha=0)$ of system (5.1) by different methods. We can see from Table 1 that our results (Corollary 3.2) are superior to those in Lemma 4 [16], Lemma 1 [4], and Theorem 1 [18].

Example 5.2. Consider the exponential stability of the neutral system (3.24) with time-varying delays and nonlinear perturbations with

$$
\begin{gathered}
A=\left[\begin{array}{cc}
-2 & 0 \\
0 & -0.9
\end{array}\right], \quad B=\left[\begin{array}{cc}
-1 & 0 \\
-1 & -1
\end{array}\right], \quad C=\left[\begin{array}{cc}
0.1 & 0 \\
0 & 0.1
\end{array}\right], \\
\beta_{1}=0.05, \quad \beta_{2}=0.1, \quad h(t)=r(t) .
\end{gathered}
$$

Decompose matrix $B$ as follows which is the same as the decomposition in Example 5.1. 
Table 2: Comparison of the maximum allowed time delay $h_{\max }$.

\begin{tabular}{lccc}
\hline Methods & $h_{\max }(\delta=0)$ & $h_{\max }(\delta=0.5)$ & $h_{\max }(\delta=0.9)$ \\
\hline Chen et al. [1] $(\alpha=0.1)$ & 1.29 & 0.94 & 0.54 \\
Our results $(\alpha=0.1)$ & 1.62 & 1.10 & 0.62 \\
\hline Chen et al. [1] $(\alpha=0.5)$ & 0.69 & 0.60 & 0.46 \\
Our results $(\alpha=0.5)$ & 0.75 & 0.61 & 0.47 \\
\hline Chen et al. [1] $(\alpha=0.7)$ & 0.57 & 0.53 & 0.43 \\
Our results $(\alpha=0.7)$ & 0.61 & 0.54 & 0.43 \\
\hline
\end{tabular}

Table 3: Comparison of the maximum allowed time delay $h_{\max }$.

\begin{tabular}{lccccc}
\hline Methods & $h_{\max }(c=0.1)$ & $h_{\max }(c=0.15)$ & $h_{\max }(c=0.2)$ & $h_{\max }(c=0.3)$ & $h_{\max }(c=0.4)$ \\
\hline Han [2] & 1.48 & 1.33 & 1.16 & 0.79 & 0.37 \\
He et al. [5] & 1.75 & 1.49 & 0.27 & 0.91 & 0.63 \\
Nian et al. [9] & 1.86 & 1.63 & 1.42 & 1.06 & 0.76 \\
Our results & 1.96 & 1.87 & 1.75 & 1.50 & 1.23 \\
\hline
\end{tabular}

Table 2 lists the comparison of the upper bounds delay for exponential stability of (5.3) by different methods. It is clear that our results (Corollary 3.3) are superior to those in Theorem $1[1]$.

Example 5.3. Consider the asymptotic stability $(\alpha=0)$ of the uncertain neutral system (4.7) with time-varying delays with

$$
\begin{gathered}
A=\left[\begin{array}{cc}
-2 & 0 \\
0 & -0.9
\end{array}\right], \quad B=\left[\begin{array}{cc}
-1 & 0 \\
-1 & -1
\end{array}\right], \quad C=\left[\begin{array}{ll}
c & 0 \\
0 & c
\end{array}\right], \quad 0 \leq c<1, \\
E=I, \quad N_{1}=N_{2}=0.2 I, \quad h(t)=r(t) .
\end{gathered}
$$

Decompose matrix $B$ as follows which is the same as the decomposition in Example 5.1.

Table 3 shows the comparison of the upper bounds delay allowed obtained from Corollary 4.2 for asymptotic stability $(\alpha=0)$ of system (4.7) with (5.4) by other methods. It can be found from Table 3 that our results are significantly better than those in $[2,5,9]$.

\section{Conclusions}

The problem of robust exponential stability for uncertain neutral systems with discrete and distributed time-varying delays and nonlinear perturbations was studied. Based on the combination of descriptor model transformation, decomposition technique of coefficient matrix, and utilization of zero equation and new Lyapunov functional, sufficient conditions for robust exponential stability were obtained and formulated in terms of linear matrix inequalities (LMIs). Numerical examples have shown significant improvements over some existing results. 


\section{Acknowledgment}

This work was supported by the Higher Education Research Promotion and National Research University Project of Thailand, Office of the Higher Education Commission, through the Cluster of Research to Enhance the Quality of Basic Education.

\section{References}

[1] Y. Chen, A. Xue, R. Lu, and S. Zhou, “On robustly exponential stability of uncertain neutral systems with time-varying delays and nonlinear perturbations," Nonlinear Analysis, vol. 68, no. 8, pp. 2464 2470, 2008.

[2] Q.-L. Han, "Robust stability of uncertain delay-differential systems of neutral type," Automatica, vol. 38, no. 4, pp. 719-723, 2002.

[3] Q.-L. Han, "A descriptor system approach to robust stability of uncertain neutral systems with discrete and distributed delays," Automatica, vol. 40, no. 10, p. 1791-1796 (2005), 2004.

[4] Y. He, Q.-G. Wang, C. Lin, and M. Wu, "Delay-range-dependent stability for systems with timevarying delay," Automatica, vol. 43, no. 2, pp. 371-376, 2007.

[5] Y. He, M. Wu, J.-H. She, and G.-P. Liu, "Delay-dependent robust stability criteria for uncertain neutral systems with mixed delays," Systems \& Control Letters, vol. 51, no. 1, pp. 57-65, 2004.

[6] O. M. Kwon, J. H. Park, and S. M. Lee, "Augmented Lyapunov functional approach to stability of uncertain neutral systems with time-varying delays," Applied Mathematics and Computation, vol. 207, no. 1, pp. 202-212, 2009.

[7] O. M. Kwon, J. H. Park, and S. M. Lee, "On delay-dependent robust stability of uncertain neutral systems with interval time-varying delays," Applied Mathematics and Computation, vol. 203, no. 2, pp. 843-853, 2008.

[8] J. H. Park, "Novel robust stability criterion for a class of neutral systems with mixed delays and nonlinear perturbations," Applied Mathematics and Computation, vol. 161, no. 2, pp. 413-421, 2005.

[9] X. Nian, H. Pan, W. Gui, and H. Wang, "New stability analysis for linear neutral system via state matrix decomposition," Applied Mathematics and Computation, vol. 215, no. 5, pp. 1830-1837, 2009.

[10] J. Tian, L. Xiong, J. Liu, and X. Xie, "Novel delay-dependent robust stability criteria for uncertain neutral systems with time-varying delay," Chaos, Solitons and Fractals, vol. 40, no. 4, pp. 1858-1866, 2009.

[11] B. Wang, X. Liu, and S. Zhong, "New stability analysis for uncertain neutral system with time-varying delay," Applied Mathematics and Computation, vol. 197, no. 1, pp. 457-465, 2008.

[12] L. Xiong, S. Zhong, and J. Tian, "New robust stability condition for uncertain neutral systems with discrete and distributed delays," Chaos, Solitons and Fractals, vol. 42, no. 2, pp. 1073-1079, 2009.

[13] Z. Zhao, W. Wang, and B. Yang, "Delay and its time-derivative dependent robust stability of neutral control system," Applied Mathematics and Computation, vol. 187, no. 2, pp. 1326-1332, 2007.

[14] F. Gao, S. Zhong, and X. Gao, "Delay-dependent stability of a type of linear switching systems with discrete and distributed time delays," Applied Mathematics and Computation, vol. 196, no. 1, pp. 24-39, 2008.

[15] P. Li, S.-M. Zhong, and J.-Z. Cui, "Stability analysis of linear switching systems with time delays," Chaos, Solitons and Fractals, vol. 40, no. 1, pp. 474-480, 2009.

[16] E. Fridman and U. Shaked, "Delay-dependent stability and control: constant and time-varying delays," International Journal of Control, vol. 76, no. 1, pp. 48-60, 2003.

[17] K. Gu, V. L. Kharitonov, and J. Chen, Stability of Time-Delay Systems,, Birkhäuser, Berlin, Germany, 2003..

[18] X. Zhu and G. Yang, "Delay-dependent stability criteria for systems with differentiable time delays," Acta Automatica Sinica, vol. 34, no. 7, pp. 765-771, 2008. 


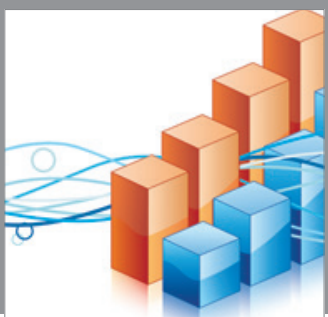

Advances in

Operations Research

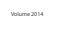

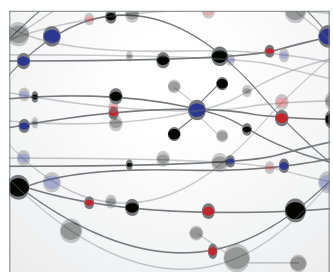

\section{The Scientific} World Journal
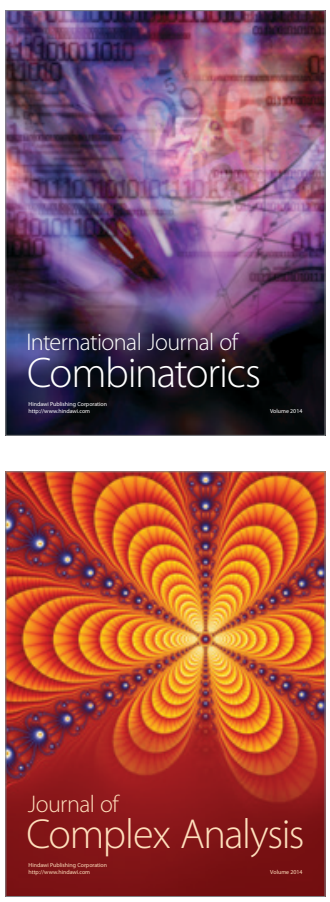

International Journal of

Mathematics and

Mathematical

Sciences
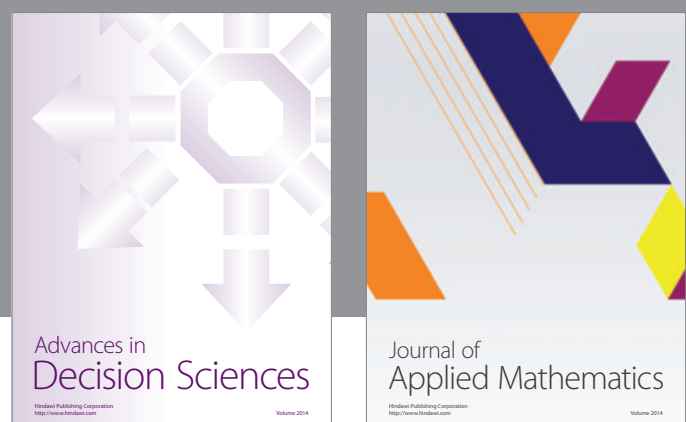

Journal of

Applied Mathematics
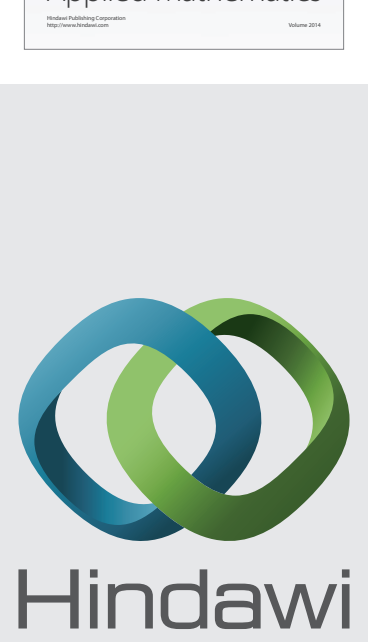

Submit your manuscripts at http://www.hindawi.com
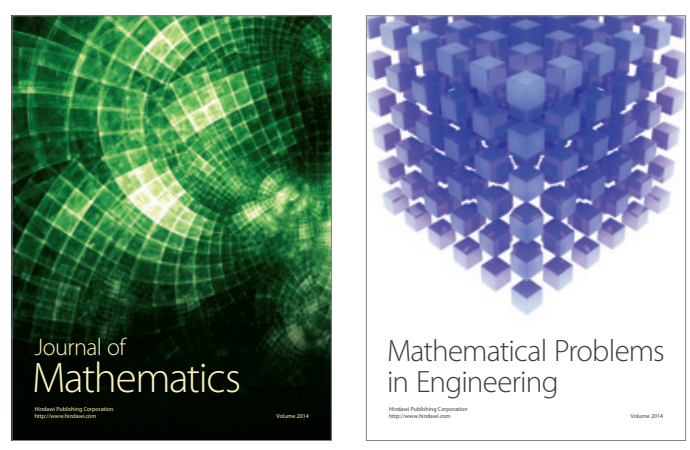

Mathematical Problems in Engineering
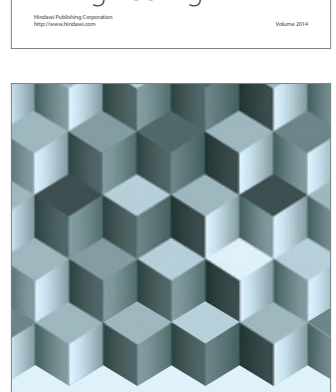

Journal of

Function Spaces
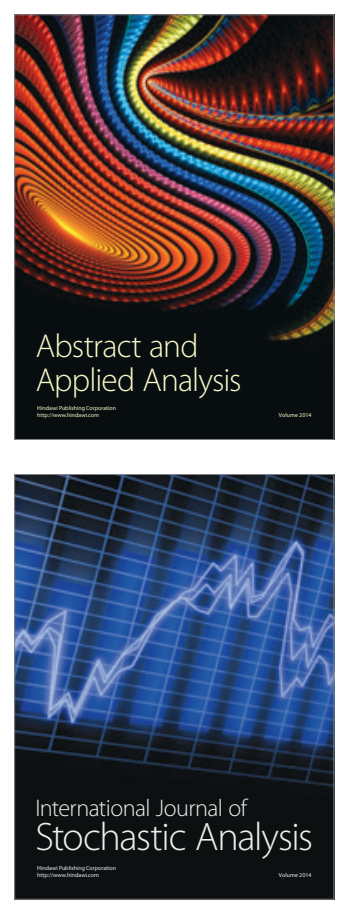

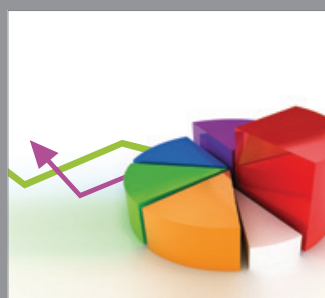

ournal of

Probability and Statistics

Promensencen
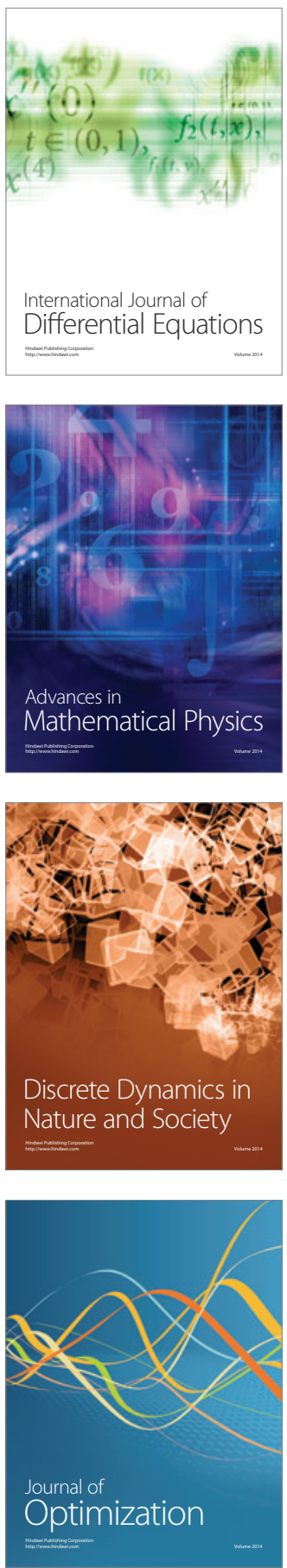